Courrier du Centre international Blaise Pascal

$4 \mid 1982$

Varia

\title{
Le manuscrit « Usage de la machine »
}

\section{Thérèse Goyet}

\section{OpenEdition}

\section{Journals}

Édition électronique

URL : http://journals.openedition.org/ccibp/405

DOI : $10.4000 /$ ccibp.405

ISSN : 2493-7460

\section{Éditeur}

Centre international Blaise Pascal

\section{Édition imprimée}

Date de publication : 4 mars 1982

Pagination : 6-13

ISSN : 0249-6674

\section{Référence électronique}

Thérèse Goyet, " Le manuscrit « Usage de la machine » », Courrier du Centre international Blaise Pascal [En ligne], 4 | 1982, mis en ligne le 25 novembre 2015, consulté le 19 avril 2019. URL : http:// journals.openedition.org/ccibp/405 ; DOI : 10.4000/ccibp.405

Ce document a été généré automatiquement le 19 avril 2019.

Centre international Blaise Pascal 


\title{
Le manuscrit « Usage de la machine »
}

\author{
Thérèse Goyet
}

1 L'extraordinaire réunion des neuf " pascalines » à l'exposition Blaise Pascal « Auvergnat » de 1981, ne sera pas, nous l'espérons, sans porter de longues conséquences. Un petit événement a jeté le CIBP, corps et biens, dans les archives de l'histoire des sciences au XVIII ${ }^{\mathrm{e}}$ siècle.

2 Tout a commencé par la curiosité avisée d'un de nos «correspondants", M. Guy Mourlevat. Depuis toujours passionné par l'évolution de la science arithmétique et l'auteur d'une analyse fouillée de La Machine arithmétique ou Pascaline (Clermont-Ferrand, CRDP, 1981, voir catalogue de la susdite exposition, p. 44), il découvrit dans les archives du musée Du Ranquet une lettre de M. Henry Malet-Daubant en date du 19 janvier 1955, et la réponse du conservateur, M. Pierre Fournier, en date du 25 du même mois. Le conservateur clermontois éludait en fait la demande posée par un memorandum du propriétaire concernant les performances possibles de la machine en sa garde, mais la correspondance nous livrait l'adresse du propriétaire du manuscrit, avec son numéro de téléphone. Et ce numéro, par un extrême bonheur, n'avait pas changé depuis 1955. Je pus donc entrer en relation avec M. Malet-Daubant au cours des vacances de Noël 1981-1982. Il s'ensuivit des tractations, menées de la manière la plus amicale, et enfin la pièce que la Bibliothèque Nationale et la société IBM avait cherché à acquérir sans jamais conclure, je l'emportai au nom du CIBP, le 18 février 1982. L'achat en a été réglé par la Ville de Clermont-Ferrand, avec une subvention exceptionnelle du Ministère de la Culture, se montant à la moitié du prix. Le manuscrit Malet-Daubant est donc maintenant la propriété de la Bibliothèque municipale de Clermont-Ferrand, qui l'a immatriculé sous le $\mathrm{n}^{\circ} 1522$ de ses manuscrits. (Voir la note publiée dans le quotidien La Montagne du 21 mai 1982).

3 La première question qu'il pose est celle de son origine. C'est la seule que nous débattrons dans ce Courrier puisque la publication critique du texte est envisagée dans un corpus réunissant documents, descriptions et explications théoriques et pratiques concernant les 
machines arithmétiques, car il nous semble que désormais le pluriel devient indispensable pour caractériser l'activité très concrète de l'inventeur - producteur.

$\mathrm{Au}$ commencement - commencement que nous arrêtons provisoirement - était l'abbé Charles Bossut. À cet abbé encyclopédiste, né à Tartaras, près de Rive-de-Gier (Loire), vers 1730, et mort à Paris le 14 janvier 1814, on est redevable de la première édition des textes scientifiques de Pascal aux t. IV et $\mathrm{V}$ de son édition des Euvres de Pascal, donnée anonymement à Amsterdam, en 1779. On a identifié sa main de façon certaine dans les corrections de notre manuscrit, mais sans pouvoir dater celles-ci. Or la chronologie relative des deux écritures, celle du lecteur Bossut et celle du copiste anonyme, nous permettrait d'approcher les conditions de l'apparition du document.

5 Les indices internes nous laisseront une grande marge pour nos conjectures. Le papier d'abord, ou plutôt les deux papiers constituant le recueil. M. Pierre Delaunay, libraire papetier à Clermont-Ferrand, d'après le filigrane propre à la copie elle-même, où l'on lit :

$\mathrm{G}$ (fleur de lys) MALMENAIDE FIN

AUVERGNE 1742

identifie le fabricant comme Genès Malmenaide, établi à Thiers entre 1750 et 1780, et il propose pour l'écriture du texte les environs de 1760. Mais la reliure (médiocre) parait être plutôt du XIXe siècle, et les pages de garde incorporées désignent comme papetier fabricant FENEROL JEUNE. L'activité des papetiers Fenerol est attestée à Chamalières en 1776, puis la famille se transporte à Thiers où un Fenerol « jeune » est attesté de 1823 à 1827, nous apprend encore M. Delaunay. C'est précisément le temps où le nouveau propriétaire - celui qui a dû faire exécuter la reliure - écrit sur une des pages de garde :

M. l'abbé Bossut me remettant cet écrit, peu de semaines avant sa mort, me dit: Cette instruction vaut beaucoup mieux que celle qui est employée dans mon édition des œuvres de Pascal, 1779, 5 vol. in-8 Mais pourquoi ne lui avez-vous pas donné la préférence ? - Parce que mon siège étoit fait. J'avais tout disposé lorsqu'on me procura cette pièce, et je ne pus l'introduire dans mon édition. " Lorsqu'en $1819 \mathrm{M}$. Lefevre, libraire fit une nouvelle et très bonne édition des œuvres de Pascal, 5 vol. in-8 je lui offris l'usage de ce cahier, il l'accepta, et sans doute l'oublia ensuite, car il ne me l'a jamais fait demander, et l'édition a paru avec l'ancienne description.

Portrait de Blaise Pascal daté du 13 e avril 1827 


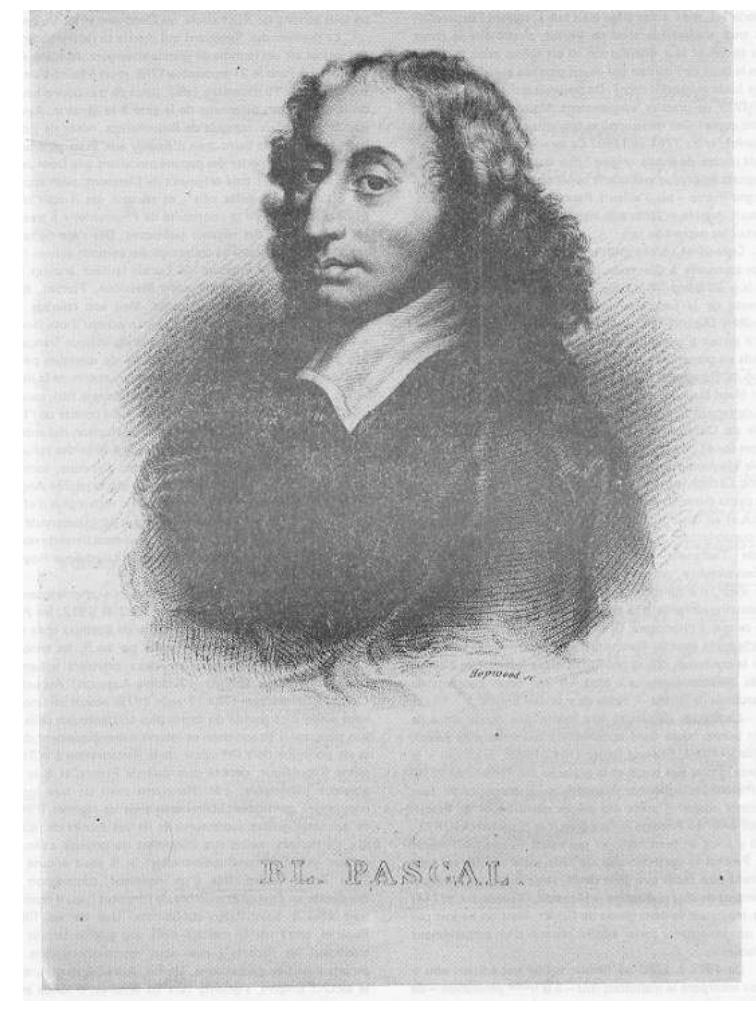

Signé Hopwood

Bossut est donc entré en possession de cette pièce trop tard " pour l'introduire dans son édition ", ce qui ne veut pas dire qu'à ce moment-là ses Euuvres de Pascal étaient déjà imprimées, mais « son siège était fait », suivant l'expression du trop académique abbé de Vertot, c'est-à-dire le choix des textes et leur distribution. Il est même assez probable que le bruit de l'édition qui devait paraître aura fait accourir vers lui le manuscrit caché. On pourrait donc dater très près de 1779 ce premier surgissement. Mais d'où venait donc cette copie ? Des milieux où se fabriquait l'Encyclopédie de Diderot, entre 1751 et 1780 ? Ce serait assez vraisemblable. Mais encore de quelle origine? Que les papiers aient été auvergnats ne prouve pas que la copie ait été effectuée en Auvergne même - où d'ailleurs Renouard qui fit relier le manuscrit semble n'avoir aucune attache - car le commerce portait les papiers au loin.

Cependant on entrevoit un rattachement avec la collection conservée à Clermont dans la famille Périer. En effet de la p. 36 à la p. 57 la copie parle d'une machine toute différente de la pascaline. En effet M. Mourlevat et $\mathrm{M}^{\mathrm{me}}$ Caroline Durand, documentaliste au CIBP, ont découvert qu'il sortait d'une pièce contenue dans un des précieux recueils en provenance de l'Oratoire de Clermont, c'est-à-dire issus de Marguerite Périer elle-même. Le copiste du manuscrit Malet-Daubant a transcrit de larges extraits de la pièce $34 \mathrm{du}$ recueil R. 1037 appartenant à la Bibliothèque municipale de Clermont-Ferrand. Il s'agit d'un opuscule de 60 pages in- $4^{\circ}$, avec 4 pages de figures. s.l.n.d., intitulé Nouvelle Machine d'Arithmétique du Sieur Grillet, Horlogeur à Paris. Le copiste a reproduit, sans porter la moindre référence, de petits éléments pourvus d'intitulés, en les mettant bout à bout et en désordre. Cela, joint aux nombreuses bourdes de sa transcription, montre bien peu de conscience professionnelle: l'acheteur, quelque «documentaliste» attaché à l' Encyclopédie, ne lirait pas de si près! Bossut, avec plus de loisir, n'a lui-même pas tout corrigé. Que l'action de transcrire remonte à la pièce clermontoise, nous l'inférons de ce 
que "l'horlogeur Grillet» devait être bien oublié un siècle après, mais un marchand quelconque pouvait faire fond sur la confusion qui se produirait entre les diverses « curiosités mathématiques » - c'est un des titres courants de l'opuscule de Grillet - tirées de si bonne origine.

L'opuscule de Grillet sera même sans doute entré de fort bonne heure dans la collection des neveux de Pascal, grâce à l'aîné, Étienne Périer (1642-1680), celui qui a le mieux connu son oncle et le préfacier des Pensées de 1670, qui avait des curiosités scientifiques. Il correspondit avec Leibniz auquel il prêta des pièces scientifiques de Pascal. C'est à lui qu'Antoine Arnauld écrit le 5 septembre 1673 : « Il y a icy un petit horloger qui ayant vû une machine de M. Paschal la perfectionnée de telle sorte qu'elle est infiniment plus facile que celle de M. votre oncle car les roues tournent de côté et d'autres. » (Arnauld, CEuvres, t. I, p. 714) On reconnaitt la description de Grillet. Mais on ne sait pas ce que les enfants Périer auront ressenti d'un surpassement si facile.

9 De 1673 à 1779 où Bossut publie son édition sans y avoir incorporé le manuscrit issu - à la $\mathrm{n}^{\text {ième }}$ génération - de l'héritage Périer, nous enregistrons la solution de continuité. Mais de la mort de Bossut jusqu'à nous, la succession est très simple à établir.

Elle se joue sur deux familles qui ont été liées par l'amitié tout au long du XIX ${ }^{e}$ siècle, les Renouard et les Vuigne.

11 Le premier des Renouard qui mérite la reconnaissance des lettres est un homme de grande envergure. Antoine-Augustin, né à Paris le 21 septembre 1765, mort à SaintValéry-Sur-Somme le 15 décembre 1853, passa de très bonne heure de la fabrication paternelle de la gaze à la librairie. Ayant épousé la fille du marquis de Beauchamps, héros de Rossbach et député de Saint-Jean d'Angély aux États généraux, il a pu entendre parler des papiers pascaliens que Dom Jean Guerrier, prieur et curé originaire de Clermont, avait apportés à l'abbaye de cette ville ${ }^{1}$, et en tout cas il était bien placé pour défendre la continuité de l'humanisme à travers les changements des régimes politiques. Dès l'âge de seize ans il avait commencé sa collection des éditions aldines : en 1795 il publie la Pharsale de Lucain (auteur à coup sûr républicain), puis successivement Massillon, Florian, Berquin, Voltaire, La Rochefoucauld. Mais son courage est aussi remarquable que son intelligence puisqu'il osa lancer «le $25^{\mathrm{me}} \mathrm{du} 1^{\mathrm{er}}$ mois l'an $2^{\mathrm{me}}$ de la république française une et indivisible », des « Observations de quelques patriotes sur la nécessité de conserver les monuments de la littérature » (Cosignataires Chardin et Charlemagne fils), suivies «le $2^{\text {me }} \mathrm{du} 2^{\text {me }}$ mois » d'une adresse « Au comité de l'Instruction publique » pour empêcher la destruction des armoiries, laquelle aurait évidemment entraîné celle des reliures et des livres eux-mêmes. Les arguments d'époque, comme de ne pas donner à enrichir la librairie de la jalouse Angleterre, eurent peut-être plus d'effet, ou du moins plus d'effet avoué, que de plus nobles considérations sur la pérennité de l'art ! Une telle intervention permit aux beaux livres de notre patrimoine de passer sains et saufs de la Bibliothèque Royale à la Nationale.

12 Antoine-Augustin Renouard a publié d'intéressantes éditions de Pascal, les Pensées en 1803 et 1812, les Provinciales en 1803. L'édition complète de Berthou chez Lefèvre en 1819 s'honore de signaler par un R. les annotations qu'elle lui doit dans ses deux premiers volumes.

13 Un des cinq enfants d'Antoine-Augustin, Augustin Charles (22 octobre 1794-17 août 1878) acquit une notoriété sinon plus grande du moins plus éclatante que celle de son père, car il fit son droit et intervint énergiquement dans la vie politique du XIX ${ }^{\mathrm{e}}$ siècle, de la 
Restauration à la Troisième République, député puis pair de France et à la fin sénateur inamovible. «M. Renouard avait de très fortes convictions politiques : libéral sous tous les régimes, il était de ces intelligences supérieures et de ces esprits de raison qui s'attachent moins aux étiquettes du pouvoir exécutif qu'aux garanties constitutionnelles ${ }^{2} »$ Il avait épousé en 1821 Adèle Girard, fille d'un ingénieur, compagnon de Bonaparte en Égypte et membre de l'institut ${ }^{3}$, qu'il rencontrait l'été à Saint-Valéry-sur-Somme. Une de ses filles, Eugénie, entra par le mariage dans une grande famille de médecins, les Richets ${ }^{4}$, que nous retrouverons dans les projets d'édition pascalienne. Un fils, Alfred-Augustin, sous le second Empire, s'orienta vers les affaires ; il fonda avec M. d'Eichtal les Salins du Midi et mourut en 1883. Mais le fils de celui-ci, Philippe-Ernest-Augustin, né le 25 octobre 1862, mort en 1934, revint, comme son arrière-grand-père au service des livres, et combien brillamment! Fondateur d'une école professionnelle de l'imprimerie, lui-même imprimeur de la Revue des Deux-Mondes, imprimeur de l'Institut, un des fondateurs de la Librairie française, il ne put tirer lui-même tout le parti de son immense érudition, appuyée sur ses propres collections, et c'est après sa mort qu'on a publié son histoire des Imprimeurs du XVI e siècle (t. 5, 1964).

Mais c'est à la bibliothèque d'Antoine-Augustin que nous nous intéressons. La Librairie Renouard, installée au début du XIX siècle, 6, rue de Tournon, en l'hôtel de Brancas, et qu'il avait cédée à son fils Jules en 1839, subsiste toujours sous le nom de Librairie Laurens. Mais sa bibliothèque personnelle, Antoine-Augustin n'a cessé de la renouveler après des ventes successives, partielles ou totales, qui ont été l'occasion, ou peut-être la conséquence, de la publication successive de catalogues, dans lesquels nous trouvons notre butin. Catalogues en 1797, 1799, 1819, 1853 ; ventes de 1804, 1820, 1821, 1825, 1828 (Londres), 1829 (Paris), 1830 (Londres), 1854, celle-ci posthume, pour laquelle une seconde édition du catalogue de 1853 est donnée par Jules-Romain Tardieu, Londres-Paris, 1854. Un Catalogue de Lettres autographes provenant du cabinet de feu M. Antoine-Augustin Renouard, ancien libraire, ancien maire du XIe arrondissement, dont la vente aura lieu le jeudi 21 juin $1855 \mathrm{et}$ jours suivants à 7 heures très précises du soir, Rue des Bons-Enfants, 28... » (Paris, Laverdet et Potin, 1855) suivit, mais celui-ci n'avait pas été rédigé par Antoine Augustin lui-même. Il contenait (p. 65, $n^{\circ}$ 544) l'annonce de deux lettres autographes de Pascal, que l'éditeur Faugère avait examinées. Mais c'est le catalogue de 1819 (4 tomes en 2 vol. ), et celui de 1853 ou 1854, qui nous parlent du manuscrit Usage de la Machine.

Le catalogue de 1819 joint à l'annonce de l'édition toute récente de Lefèvre (1818 (sic), 5 vol. in-8. Gr. pap. vélin), un commentaire qui confirme, et élargit la note de 1827 que nous avons citée : 


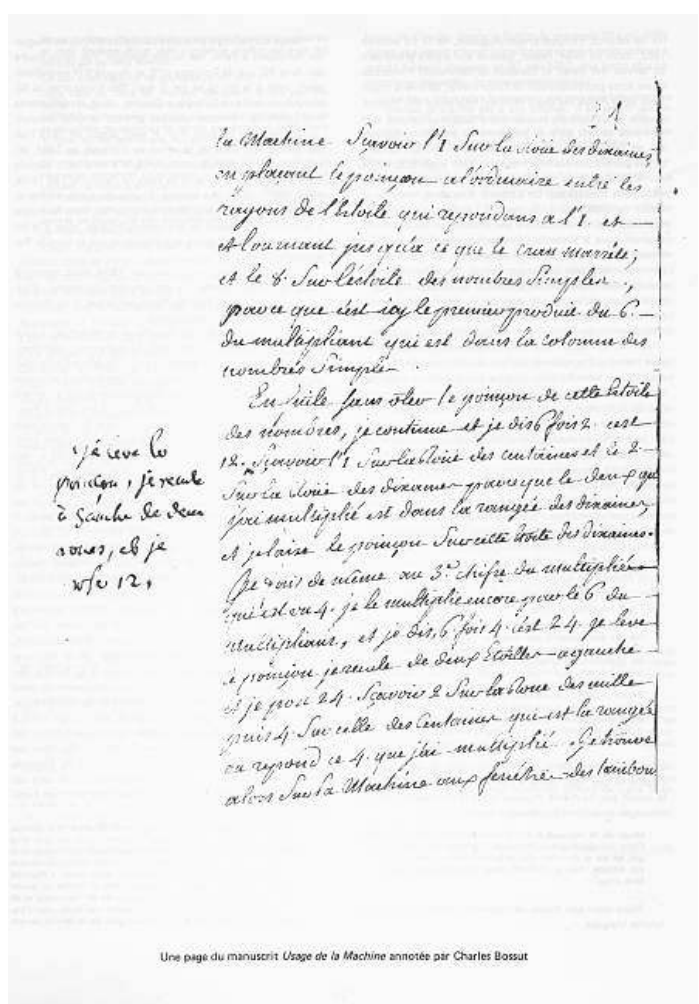

Peu de semaines avant sa mort, le savant Bossut vint me voir, et me dit que puisqu'à son exemple j'avais donné des soins à l'exacte réimpression des ouvrages de Pascal, il voulait qu'après lui je restasse possesseur du peu de papiers qu'il avait de ce grand homme; et il me remit une lettre de Pascal, deux autre à lui adressées par Bourdelot, et par un savant de Liège, une de Fermat à Carcavi, et une description de la machine arithmétique, meilleure, me dit-il que celle des Euvres complètes. J'ai joint ces diverses pièces à cette nouvelle édition, avec un feuillet de la main de l'abbé Bossut, contenant un passage de l'éloge de Pascal qui n'avait pu être imprimé 5 .

"Ces diverses pièces » étant au nombre de six, c'est la cinquième que nous avons à suivre à la piste. Renouard ne la rendit pas lui-même puisque nous la retrouvons dans le catalogue de la vente posthume :

Usage de 1a Machine arithmétique de Pascal. Manuscrit in-4. Cette instruction, non imprimée, me vient de l'abbé Bossut, qui, en me la donnant, me dit qu'elle valait mieux que celle de son édition, mais qu'il l'avait reçue trop tard pour pouvoir en faire usage ${ }^{6}$.

Nous voici aux mains de l'acheteur, c'est-à-dire dans la famille Vuigner.

18 Nous connaissons plusieurs domiciles d'Antoine-Augustin Renouard à Paris, rue SainteApolline, rue Saint-André des Arcs 42, rue de Tournon n ${ }^{\circ}$ 6, et dans le $\mathrm{XI}^{\mathrm{e}}$ arrondissement, mais à la fin de sa vie, il avait fait transporter sa bibliothèque à Saint-Valéry-sur-Somme, dans les bâtiments subsistants de l'antique abbaye, et c'est là qu'il mourut.

Ce domaine lui était cher. Il avait acquis en 1819 l'un des quatre lots issus de la vente de l'abbaye en 1791, et, avec une patience probablement égale à celle qu'il mettait à ses collections de livres, il ne cessa de l'arrondir: en 1819, 1823, 1827, 1836. Ses enfants, Charles-Augustin, et Marie Eugénie Ricot-Renouard, imitèrent cette politique favorable à 
la réunion de toutes les branches familiales, mais à la fin il fallut sortir de l'indivis et l'abbaye quitta la famille Renouard en 1859.

Notre manuscrit vendu, lui, en 1854, était revenu à Saint-Valéry-sur-Somme où son nouveau possesseur, d'ailleurs apparenté à la famille Renouard grâce à de doubles liens d'alliance, était bien enraciné.

21 Là aussi la famille est vigoureuse et inventive. LouisAndré-Noël Vuigner (1770-1848, 10 septembre), commandant d'artillerie sous l'Empire, a pour fils Marie-Émile Vuigner, né en 1798 à Saint-Valéry-sur-Somme, mort au même lieu le 7 novembre 1865. La Restauration ayant supprimé l'emploi de son père, Marie-Émile renonça à entrer à Polytechnique et il semble s'être formé tout seul. Son grand-père maternel, Masset, «avait déterminé par ses démarches énergiques l'ouverture du canal de la Somme en 1786 »; son parent ${ }^{7}$, l'ingénieur en chef des ponts et chaussées, Pierre Simon Girard, avait commencé les travaux du canal de l'Ourcq. Marie-Émile Vuigner fut chargé des travaux du canal de l'Ourcq, des canaux Saint-Martin et Saint-Denis. Il construisit les magasins généraux de La Villette, et, pour les chemins de fer de l'Est, la ligne de Paris à Meaux et celle de Paris à Mulhouse, et il eut une part très importante dans la construction du pont de Strasbourg à Kehl. Son fils Henri Louis (28 novembre 1835, 10 mai 1903), et son petitfils, Jean-Baptiste-Marie-Émile (10 octobre 1866, 3 septembre 1951) furent tous deux ingénieurs civils des mines, et c'est de son arrière-petite-fille Alice, Madame MaletDaubant, que le CIBP a reçu le manuscrit Usage de la Machine.

Lequel de ses ancêtres l'avait acheté à la famille Renouard ? Certainement Marie-Émile, le constructeur du pont de Kehl, à la vente de 1854. Il avait aussi dans sa bibliothèque, mais nous ne savons pas la provenance, la Première lettre à un Provincial, du 23 janvier 1656; la seconde édition des Pensées de Pascal, de 1670, et les Traités de l'Équilibre des Liqueurs, de 1648. Un mémoire de la main de Madame Émile Vuigner, née Hortense Rousselin, à la date du samedi 11 avril 1885, nous a conservé une tradition familiale sur le motif de l'achat :

L'édition des œuvres de Pascal en 5 volumes in.8 (probablement celle de l'abbé Bossut $^{8}$ ) ayant atteint un prix trop élevé, $\mathrm{M}^{\mathrm{r}}$ Émile Vuigner a acheté l'instruction sur l'usage de la machine à calculer, manuscrite sur un cahier cartonné, pensant que le portrait très bien gravé de Pascal ferait plaisir à Madame Émile Vuigner qui désirait conserver dans la famille un exemplaire des œuvres de Pascal en souvenir de $\mathrm{M}^{\mathrm{r}}$ Renouard et de sa complaisance à lire et même à prêter ses livres pour l'instruction ou l'amusement des jeunes gens de la famille venant passer quelques jours de leurs vacances à Saint-Valery chez lui et chez Madame Ricot dans l'ancienne abbaye qui avait été achetée par les deux familles.

Ce qui avait ranimé ce souvenir, c'est que :

Dans l'été de $1884 \mathrm{M}^{\mathrm{r}}$ Philippe Renouard, arrière-petit-fils de $\mathrm{M}^{\mathrm{r}}$ Augustin Renouard, étant venu à Saint-Valery avec $\mathrm{M}^{\mathrm{me}}$ Alfred Renouard sa mère, pour visiter l'ancienne Abbaye et renouveler les souvenirs de famille en a entretenu $\mathrm{M}^{\mathrm{me}}$ Émile Vuigner qui est rentrée avec eux dans cette ancienne Abbaye et dans son passé.

$\mathrm{M}^{\mathrm{r}}$ Henri Vuigner ayant retrouvé dans sa bibliothèque le manuscrit contenant l'instruction sur l'usage de la machine à calculer de Pascal a proposé à $\mathrm{M}^{\mathrm{me}}$ Émile Vuigner de l'offrir à $\mathrm{M}^{\mathrm{r}}$ Philippe Renouard en souvenir de son arrière-grandpère, et aussi pour faire entrer cette instruction dans une édition des œuvres de Pascal, s'il s'en imprime une nouvelle, dans l'intérêt du mérite de cette édition.

Henri Vuigner remit bien le manuscrit à Philippe Renouard, chez sa mère, rue de Beaune, $\mathrm{n}^{\circ}$ 6, le 11 avril 1885, mais ce n'était qu'un prêt. En de si bonnes mains toutefois cela fut suffisant pour attirer sur lui l'attention des érudits intéressés par la recherche des textes 
de Pascal. Philippe Renouard le fit voir à Brunetière et leur conclusion fut « ou ce manuscrit aura été composé pour Diderot qui en aura trouvé une autre version meilleure, ou il provient de Clermont ${ }^{9}$ ». Philippe Renouard le montra aussi à son cousin, le $\mathrm{D}^{\mathrm{r}}$ Charles Richet, qui le communiqua à Charles Henry, bibliothécaire à la Sorbonne, et celuici en écrivit au Bulletino di bibliografia, à Rome, en $1887^{10}$.

Charles Buloz, Directeur de la Revue des Deux Mondes, ayant épousé la fille du Docteur Richet, il était de la famille Charles Henry lui écrit «Votre manuscrit » à la date du 9 juin 1883, et il conclut :

À mes yeux ce document est très important et indispensable pour la prochaine édition si désirée des œuvres mathématiques de Pascal. [...] Il me paraît impossible de prouver que cette pièce n'est pas de Pascal.

Ce prochain éditeur serait-il l'illustre Ernest Havet? Madame Émile Vuigner paraît l'avoir espéré puisqu'elle lui fit porter le manuscrit par son fils Henri Vuigner, avec une copie de la lettre de Charles Henry à Buloz, le vendredi 22 avril 1887. Havet, qui venait d'avoir " une longue et grave indisposition », écrivit à Henri Vuigner, le 20 mai 1887, une lettre plus circonspecte que celles de ses correspondants:

J'ai beau lire et relire le manuscrit que vous avez bien voulu me confier, je n'arrive pas à une solution. Il est certain que cet écrit est de l'auteur d'une machine arithmétique, or ni dans la langue ni dans le ton général, il n’y a rien qui me paraisse empêcher de croire que cet auteur est Pascal lui-même.

S'agirait-il du « Discours » que Pascal avait adressé à Bourdelot à l'intention de la reine Christine de Suède? Mais ce qui troublait le plus Havet, c'est que Diderot dans sa description, au $\mathrm{t} 1^{\text {er }}$ de l'Encyclopédie, de la machine de Pascal ne parle « ni de l'extraction des racines carrées et cubiques, ni de la pièce de sa machine qui servait à ces deux opérations ». La marche à suivre pour Havet serait : collationner le texte même de Diderot avec celui que Bossut reproduit dans son édition de Pascal ; rapprocher de la description du manuscrit celle que Diderot évoque, publiée par M. de Lépine en 1725 ; enfin examiner à fond la machine de Pascal exposée au Conservatoire des Arts et Métiers. (Havet paraît n'en pas connaître d'autre.)

Ce programme était la sagesse même. Seulement Havet mourut en 1889 et notre Usage de la Machine manqua, pour la troisième fois, l'occasion d'être édité.

Des éléments souhaités par Havet nous disposons aujourd'hui avec une surabondance que le vieux critique n'imaginait pas. Le CIBP a tenu réunies pendant un mois toutes les machines connues de la fabrication de Pascal. Deux restent dans son voisinage, les autres lui répondent par leurs photographies. Et surtout il a fait entrer en collaboration le temps, qui fait la lumière dans l'histoire pour ceux qui l'achètent par la patience, et les personnes qui forment sur les questions arrivées à maturité autant de rayons vivants en convergence. 
Abbaye de Saint-Valéry-sur-Somme.

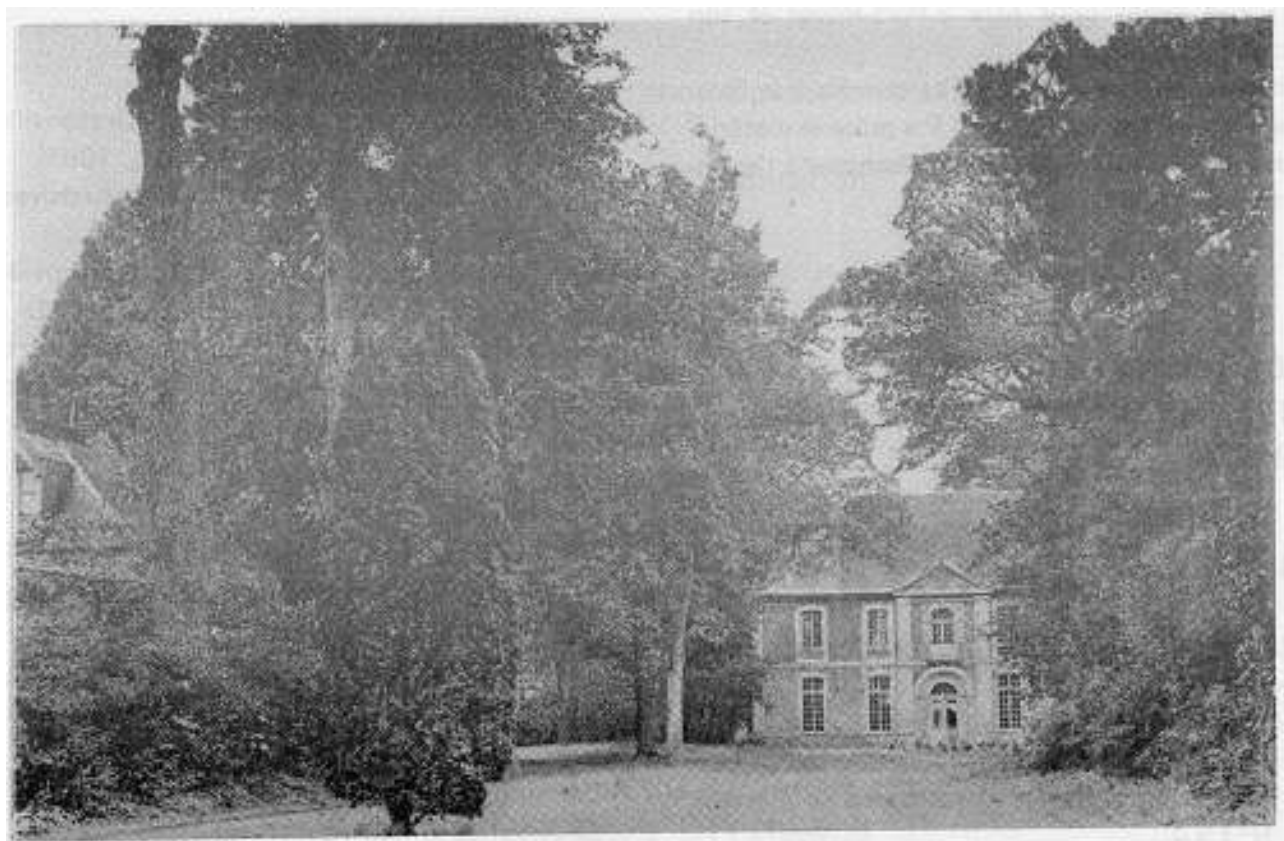

La maison conventuelle. XVIII siècle (D'après une carte postale)

\section{Vue panoramique de l'Abbaye}

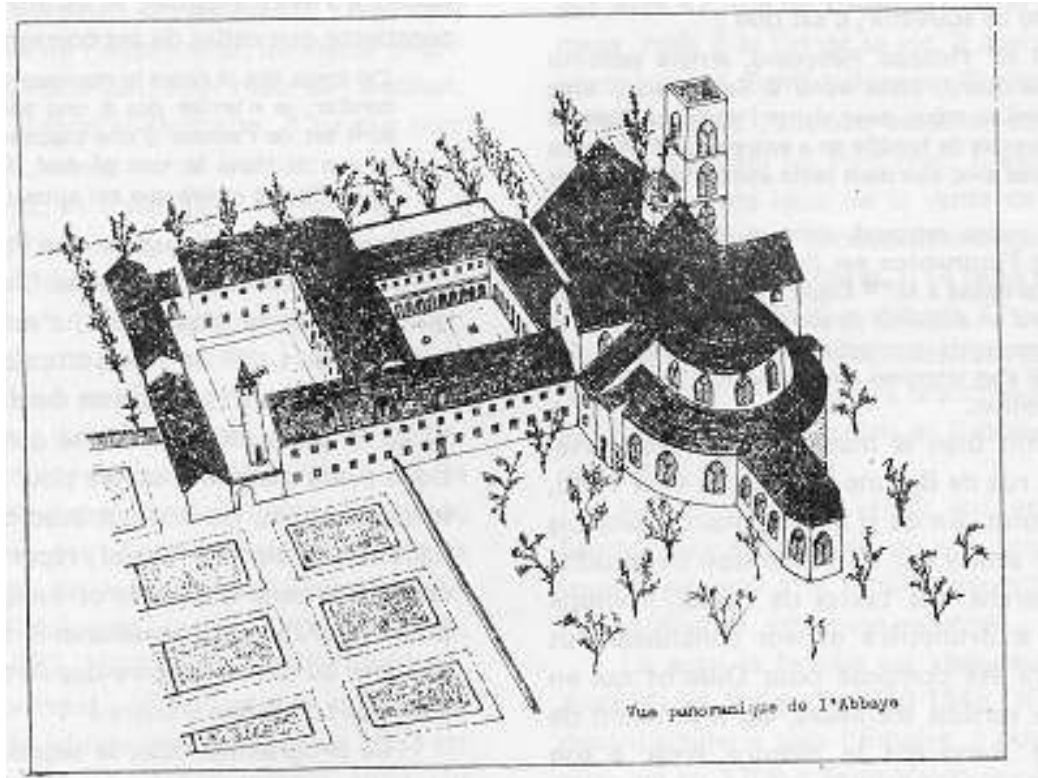

L'Abbaye de Saint-Valéry-sur-Somme, en 1791. (Dessin de $M^{\text {me }}$ Deloison Joanna, dans Histoire de l'ancienne abbaye de Saint-Valéry-sur-Somme, 1981). 


\section{BIBLIOGRAPHIE}

\section{Manuscrits}

- Usage de la machine - [1760] ca. A - K ff. bl. + L + 69 p. + [6] ff. bi. + 1 f. de portrait. Bibliothèque Municipale de Clermont-Ferrand, Ms 1522.

- Archives Malet-Daubant. CIBP.

Imprimés

- Recueil factice réunissant outre l'ensemble de l'œuvre mathématique de Pascal et une partie de son œuvre physique, diverses pièces scientifiques du XVII ${ }^{\mathrm{e}}$ siècle en rapport avec celle-ci.

Bibliothèque Municipale de Clermont-Ferrand, R. 1037 (pour la pièce 34).

Arnauld (Antoine). Lettre à Étienne Périer du 5 septembre 1673, dans Euvres, Lausanne 1775 (réimpression), p. 714-715.

Euvres de Blaise Pascal [publiées par l'abbé Charles Bossut]. La Haye : Detune [Paris : Nyon], 1779. -5 vol. in- $8^{\circ}$ : portrait gravé d'après L.-N. Quesnel, pl. gr.

Euvres de Blaise Pascal. Nouvelle édition [par Berthou]. Paris, Lefèvre, 1819, 5 vol. in-8º pl. gr.

RENOUARD Antoine-Augustin. Catalogue de la bibliothèque d'un amateur, Paris, 1819, 4 t. en 2 vol. B. N. : Q. 8340-8341.

TARDIEU Jules-Romain. Nécrologie. M. Antoine Augustin Renouard. Paris, 1854 , in- $8^{\circ}, 4$ p. B. N. : $8^{\circ}$ Ln ${ }^{27} .17261$.

[TARDIEU Jules-Romain] Catalogue d'une précieuse collection de livres, manuscrits, autographes, dessins et gravures composant la bibliothèque de M. Antoine Augustin Renouard, Ancien libraire, ancien maire du $\mathrm{XI}^{e}$ arrondissement. Dont la vente aura lieu le Lundi 20 novembre et les trente jours suivants, à 7 heures précises du soir, Rue des Bons-Enfants, 28, Maison Sylvestre [...] Paris-Londres, 1854, 420 p. B.N. : 8016.

MICHAUD, Biographie universelle ancienne et moderne, t. 35 article RENOUARD (par Gustave Brunet) PERDONNET A. Notice sur Émile Vuigner, ingénieur civil. - Extrait des Mémoires de la Société des Ingénieurs civils. Paris, 1866 , in- $8^{\circ}, 8 \mathrm{p}$.

PICOT Georges. Notice historique sur la vie et les travaux de Charles Renouard. Paris, Institut de France, 1901 , in $-4^{\circ}, 55 \mathrm{p}$.

MAIRE Albert. Bibliographie générale des œuvres de Blaise Pascal. t. I, Pascal savant, p. 66. Paris, 1925.

TATON René. «Sur l'invention de la machine arithmétique », dans L'Euvre scientifique de Blaise Pascal, p. 226, 228. Paris, 1964 , in- $8^{\circ}, 310$ p. (Textes publiés auparavant dans la Revue d'Histoire des Sciences, t. XV. 1962 et t. XVI, 1963)

Imprimeurs et libraires parisiens du XVI ${ }^{e}$ siècle.

Ouvrage publié d'après les mss. de Philippe Renouard, par le service des Travaux historiques de la Ville de Paris, avec le concours de la Bibliothèque nationale. t. I Notice de Pierre Marot. Paris, 1964. 
MESNARD Jean dans Blaise Pascal Euvres complètes, t. II, p. 318, n. 2. Paris, 1970, in-16, 1345 p.

[Marie-Louise Patenôtre-Bernard], Histoire de l'ancienne Abbaye de Saint-Valéry-sur-Somme de 1791 à nos jours, Bulletin $\mathrm{n}^{\circ} 12$, de la Société d'Archéologie et d'Histoire de Saint-Valéry-sur-Somme, du Ponthieu et du Vimeu, Saint-Valéry-sur-Somme, 1981, 56 p.

\section{NOTES}

1. «Afin de ne rien perdre de ce qui venait de la bibliothèque de Mademoiselle Périer, Dom Guerrier fit faire des recueils d'une infinité de brochures et de feuilles volantes, et autres écrits qui se trouvaient parmi les livres ». [Dom Tassin] Histoire littéraire de la Congrégation de Saint-Maur, Paris, 1770. in-4. Dans Blaise Pascal. CEuvres complètes, p.p. Jean Mesnard, t. I (1964), p. 1175.

2. Georges Picot, Notice..., 1901, p. 51.

3. Pierre-Simon Girard, Caen, 4 novembre 1765, Paris, 21 novembre 1835.

4. Mariage d'Eugénie Renouard et du $\mathrm{D}^{\mathrm{r}}$ Richet, en l'église de la Madeleine, le 14 juillet 1849. Le chirurgien. Alfred Richet (Dijon 1816 - Hyères 1891) est le père du physiologiste Charles Richet (1850-1935), père de Charles Richet, autre physiologiste (1882-1966).

5. Antoine-Augustin Renouard, Catalogue..., 1918, t. 3, p. 296.

6. Catalogue d'une précieuse collection de livres..., Paris Londres, 1854. Section Belles-Lettres, p. 234, n - 2359. En marge, à 1'encre, cette note : $8 \%$, indication relative à la vente effective probablement.

7. Nous n'avons pas découvert le degré de parenté entre Vuigner et Girard. Nous en croyons la notice de Perdonnet. C'est le second lien avec la famille Renouard, par le mariage d'Augustin Charles avec Adèle Girard. Le premier remontait à l'ancêtre Masset.

8. Nous pensons plutôt qu'il s'agissait de celle de Lefèvre, citée plus haut, sur grand papier, avec ou sans quatre des cinq pièces adjointes, le manuscrit Usage ayant été disjoint de toute façon.

9. Lettre de Philippe Renouard à Henri Vuigner, Montpellier, 29 avril 1885.

10. Reproduit dans Maire, Bibliographie..., 1925, t. I, p. 66.

INDEX

Mots-clés : Pascal, usage de la machine, pascaline

\section{AUTEUR}

THÉRÈSE GOYET

Professeur à l'Université II de Clermont-Ferrand 